\title{
Les constructions partitives pronominales en français : une analyse de corpus ${ }^{1}$
}

\author{
Tremblay, Mireille \\ Université de Montréal \\ mireille.tremblay.4@umontreal.ca
}

Résumé. Contrairement aux autres langues romanes, le français exige la présence de la préposition entre dans les constructions partitives pronominales: plusieurs d'entre nous; *plusieurs de nous. Le présent article poursuit deux objectifs : 1documenter les contextes qui favorisent ou exigent la présence de la préposition entre dans ces constructions pronominales et 2- fournir une analyse quantitative de la variation. Pour ce faire, nous avons procédé à l'analyse comparative de deux corpus de journaux : le journal Le Monde 2002 et un corpus de journaux canadiens de la même époque. Notre analyse quantitative permet de distinguer les déterminants qui sélectionnent d'entre de façon catégorique ou quasi-catégorique des déterminants qui permettent la variation entre de et d'entre et de montrer l'existence d'un effet lexical significatif dans le choix de la variante, et ce, dans les deux corpus.

\begin{abstract}
Unlike other Romance languages, French requires the presence of preposition entre "between" in partitive pronominal constructions: plusieurs d'entre nous vs. *plusieurs de nous "many of us". The goal of this paper is twofold: 1- to document the contexts that favor or require the presence of preposition entre in pronominal constructions, and 2- to provide a quantitative analysis of the variation. In order to do this, I conducted a comparative analysis of two newspaper corpora: the French newspaper Le Monde 2002 and a corpus of Canadian newspapers from the same period. The quantitative analysis shows a distinction between determiners which (quasi-)categorically require d'entre and determiners which allow variation between de and d'entre. The analysis shows the existence of a significant lexical effect in the choice of the variant and this in both corpora.
\end{abstract}




\title{
1 Introduction
}

En anglais (1) et en espagnol (2), les pronoms du pluriel apparaissent nus lorsque précédés d'un quantificateur comptable.
(1) a.two of us
b. many of us
c. how many of us ?
d.none of us
(2) a. dos de nosotros
b. muchos de nosotros
c. ¿cuántos de nosotros?
d. ninguno de nosotros

En français, les pronoms du pluriel semblent s'inscrire dans une logique différente, puisqu'ils permettent très difficilement les constructions en (3). Les mêmes exemples deviennent toutefois parfaitement acceptables lorsque la préposition entre est insérée devant le pronom (4).
(3) a. \#deux de nous
b. \#plusieurs de nous
c. \#combien de nous
d. \#personne de nous
(4) a.deux d'entre nous
b.plusieurs d'entre nous
c. combien d'entre nous
d.personne d'entre nous

Le contraste entre les exemples en (3) et (4) a d'ailleurs été noté par Franckel \& Paillard, qui l'attribuent au fait que les pronoms du pluriel désignent un ensemble non différencié. Selon ces auteurs, la préposition entre aurait donc pour fonction de partitionner l'ensemble dénoté par le pronom.

\begin{abstract}
«Cela montre que les pronoms nous/vous/eux/elles désignent un ensemble qui en tant que tel, est incompatible avec une partition. Dans ces exemples, entre a pour fonction de différencier l'ensemble auquel réfère le pronom, et donc de le rendre quantifiable.»
\end{abstract}

(Franckel \& Paillard 2007:48)

Mélis (2003) note toutefois que trois contextes permettent la variation entre les formes d'entre et de: aucun, chacun et un. Pour Mélis, l'alternance d'entre/de serait plutôt syntaxiquement conditionnée. 


\begin{abstract}
«La seconde préposition a aussi un statut particulier dans le cas de d'entre servant à introduire les compléments de certains pronoms et qui alternent régulièrement avec le simple de après aucun, chacun et un. Si cette suite forme une préposition complexe, c'est non pour des raisons sémantiques mais pour des raisons syntaxiques, puisqu'elle apparaît comme la variante conditionnée de la préposition de.»
\end{abstract}

(Mélis $2003: 121-122$ )

Le présent article poursuit deux objectifs : 1- documenter les contextes qui favorisent ou exigent la présence de la préposition entre et 2- fournir une analyse quantitative de la variation d'entre/de. Pour ce faire, nous avons procédé à l'analyse comparative de deux corpus de journaux : le journal Le Monde 2002 et un corpus de journaux canadiens de la même époque. Notre discussion est organisée de la façon suivante. La section 2 présente le cadre méthodologique de la présente étude. La section subséquente est dévolue aux résultats des analyses distributionnelles et distingue les contextes qui exigent la présence de la préposition entre des contextes qui permettent la variation. La section 4 discute des résultats obtenus à la section précédente et présente quelques pistes d'analyse. Après cette discussion, nous concluons en résumant les principales généralisations confirmées par notre analyse comparative, tout en en reconnaissant les limites, et proposons de nouvelles pistes de recherche.

\title{
2 Méthodologie
}

La section 2.1 présente brièvement le corpus que nous avons utilisé. Par la suite, nous décrivons le processus de codification des données (section 2.2) et les analyses statistiques que nous avons effectuées (section 2.3).

\subsection{Corpus}

La présente étude repose sur l'analyse de données journalistiques extraites de deux sous-corpus du corpus Le Migou. Le Migou est un concordancier Web qui permet la consultation des corpus généraux et spécialisés multilingues de l'Observatoire de linguistique Sens-Texte (OLST) de l'Université de Montréal. Les deux sous-corpus sélectionnés sont les suivants : le sous-corpus du journal français $L e$ Monde 2002 (31 354097 occurrences) et le sous-corpus de presse canadien (8 256841 occurrences) de la même année. Le sous-corpus canadien est composé d'extraits de journaux de trois provinces canadiennes : L'Acadie nouvelle de Caraquet au Nouveau-Brunswick, Le Droit d'Ottawa en Ontario et, au Québec, Le Nouvelliste de Trois-Rivières, La Presse de Montréal, Le Quotidien de Saguenay, Le Soleil de Québec et La Tribune de Sherbrooke.

Nous avons considéré deux contextes : 1-de+nous/vous/eux/elles et 2-entre+nous/vous/eux/elles, pour un total de 7471 occurrences. Toutes les constructions non partitives ont été exclues. Nous avons donc éliminé toutes les formes où de nous/de vous/d'eux/d'elles ou entre nous/entre vous/entre eux/entre elles occupaient la fonction de complément d'un nom, d'un verbe ou d'un adjectif (5a-b). Tous les pronoms clitiques nous et vous suivis d'un infinitif (5c) ont aussi été éliminées.

(5) a. N/V/A de nous/vous

b. N/V/A entre nous/vous

c. de nous/vous + INF

Au final, nous avons dénombré un total de 3961 occurrences (3 254 du sous-corpus Le Monde 2002 et $\mathbf{7 0 7}$ du sous-corpus de presse canadien). 


\begin{tabular}{|l|l|l|l|l|}
\hline & \multicolumn{2}{|c|}{ Le Monde 2002 } & \multicolumn{2}{c|}{ Presse canadienne } \\
\hline & $\mathrm{N}$ & $\%$ & $\mathrm{~N}$ & $\%$ \\
\hline partitives & 3252 & 57,8 & 707 & 38,8 \\
\hline exclusions & 2375 & 42,2 & 1137 & 61,7 \\
\hline Total & 5627 & & 1844 & \\
\hline
\end{tabular}

Tableau 1: Formes retenues ou exclues

\subsection{Codification}

Dans un premier temps, chaque construction partitive a été codée en fonction des critères suivants : 1présence/absence de entre, 2- type de déterminant et 3- personne du pronom pluriel. Le tableau 2 résume la catégorisation retenue.

\begin{tabular}{|c|c|c|c|}
\hline \multicolumn{5}{|c|}{ Variable dépendante } \\
\hline \multicolumn{5}{|c|}{ Type de déterminant } \\
\hline \multicolumn{2}{|c|}{ absence de entre } \\
\hline démonstratif & nombre cardinal & nombre ordinal & pourcentage \\
\hline superlatif & Mot Qu & quantificateur & fraction \\
\hline \multicolumn{4}{|c|}{ Personne } \\
\hline \multicolumn{2}{|c|}{2 : vous } & $3:$ eux/elles \\
\hline
\end{tabular}

Tableau 2: Analyses principales

Nous avons ainsi distingué huit types de déterminants, exemplifiés en (6).

(6) a. ceux d'entre eux

b. deux d'entre eux

démonstratif

c. le deuxième d'entre eux

nombre cardinal

d. $2 \%$ d'entre eux

e. les meilleurs d'entre eux

nombre ordinal

f. combien d'entre eux

pourcentage

g. plusieurs d'entre eux

superlatif

mot Qu-

quantificateur

h. deux tiers d'entre eux

fraction

Pour des raisons que nous expliquons à la section 3.3, nous avons par la suite procédé à des analyses secondaires : type lexical du quantificateur, contexte syntaxtique du numéral un(e), personne et genre des constructions aucun(e), chacun(e) et l'un(e).

\subsection{Analyses statistiques}

Les deux sous-corpus ont été analysés séparément. L'effet des facteurs linguistiques a été analysé à l'aide du programme GoldVarb Lion (Rand \& Sankoff 1990; Sankoff et al. 2005), un logiciel de statistiques qui réalise des analyses de régression logistique multivariées sur plusieurs facteurs en s'appuyant sur l'estimation de vraisemblance maximale pour une moyenne pondérée d'un échantillon donné et exprime la probabilité d'apparition de la variable dépendante. Goldvarb fournit trois types d'indices (Poplack \& Tagliamonte 2001): 1- la signification statistique d'un facteur, avec un seuil de .05; 2- 1'effet du facteur 
mesuré par le poids de ce dernier; 3- la direction de l'effet telle que mesurée par la force relative de chaque groupe de facteurs.

Aux fins de la présente étude, la variable dépendante est la présence/absence de entre. La variante d'aplication pour les analyses effectuées est la présence de entre; en d'autres termes, nos analyses présentent le pourcentage des pronoms précédés par la forme complexe d'entre (plutôt que le pourcentage de pronoms précédés simplement par de).

\section{Résultats}

\subsection{La distribution globale}

La tableau 3 présente la distribution globale des deux variantes, de et d'entre, en fonction du type de déterminant. Comme nous l'avons mentionné précédemment, les deux sous-corpus ont été analysés séparément.

\begin{tabular}{|c|c|c|c|c|c|c|}
\hline & \multicolumn{3}{|c|}{ Le Monde 2002} & \multicolumn{3}{|c|}{ Presse canadienne } \\
\hline & Entre & & Total & Entre & & Total \\
\hline DÉTERMINANT & $\mathrm{N}$ & $\%$ & & $\mathrm{~N}$ & $\%$ & \\
\hline Démonstratifs & 37 & $100 \%$ & 37 & 4 & $100 \%$ & 4 \\
\hline Nombres ordinaux & 80 & $100 \%$ & 80 & 2 & $100 \%$ & 2 \\
\hline Pourcentages & 262 & $100 \%$ & 262 & 54 & $100 \%$ & 54 \\
\hline Fractions & 47 & $100 \%$ & 47 & 3 & $100 \%$ & 3 \\
\hline Superlatifs & 145 & $100 \%$ & 145 & 11 & $91,7 \%$ & 12 \\
\hline Mots QU- & 17 & $89,5 \%$ & 19 & 6 & $75 \%$ & 8 \\
\hline Quantificateurs & 1264 & $89,9 \%$ & 1406 & 328 & $87,2 \%$ & 376 \\
\hline $\begin{array}{l}\text { Nombres } \\
\text { cardinaux }\end{array}$ & 803 & $61 \%$ & 1324 & 169 & $67 \%$ & 251 \\
\hline & 2591 & & 3254 & 574 & & 707 \\
\hline
\end{tabular}

Tableau 3: Distribution des variantes de et d'entre dans les constructions partitives pronominales

On observe très peu de variation entre les deux sous-corpus, ce qui tend à appuyer la thèse qu'il existe très peu de différence dans le français écrit des deux côtés de l'Atlantique. La section 3.2 traite des déterminants qui sélectionnent d'entre de façon obligatoire ou quasi obligatoire, alors que les déterminants qui permettent la variation sont abordés aux sections 3.3 et 3.4.

\subsection{Les contextes (quasi) obligatoires}

Dans les deux sous-corpus, nos résultats montrent la régularité du patron décrit en (3) et (4): 1'utilisation de la préposition entre devant les pronoms du pluriel est systématique avec les démonstratifs, les nombres ordinaux et les pourcentages. Elle est quasi obligatoire dans le cas des superlatifs et des mots Qu-. 
Le seul cas de superlatif sans entre provient du corpus canadien. Il s'agit d'un cas de discours rapporté qui implique une construction particulière où le pronom est modifé par le nombre cardinal deux.

(7) Richard et son coéquipier Benoît Labrosse auraient pu intervenir, car les deux ont des connaissances en réanimation. « Si c est Laurent qui y est allé, c'est qu'il est le meilleur nageur de nous deux. C'était donc préférable qu'il se porte au secours de Raymond, car on gagnait du temps », racontait Labrosse. Le Nouvelliste

Les trois exceptions impliquant des déterminants Qu- sont données en (8), (9) et (10). Deux de ces exceptions proviennent du journal Le Monde, alors que la troisième est issue du sous-corpus canadien.

(8) Qui de nous ne pense à cette heure à l'apparition de Renoir au premier acte de Siegfried, frappant les talons pour saluer Geneviève?

Le Monde 2002

(9) Ça vous intéresserait de savoir duquel de vous trois il s'agit?

Le Monde 2002

(10) Lequel de nous deux est le plus normal ?

L'Acadie Nouvelle

On peut noter encore une fois la présence d'un modificateur cardinal en position post-pronominale dans deux des trois exceptions. Nous discuterons du rôle syntaxique de ces modificateurs cardinaux postnominaux à la section 4.1 .

\subsection{Les contextes variables}

Le tableau 3 met en évidence le fait que deux types de déterminants permettent la variation : les quantificateurs et les nombres cardinaux, et ce, dans des proportions semblables dans les deux souscorpus. Afin de mieux décrire ces deux domaines d'application de la variable, nous avons procédé à des analyses secondaires.

\subsubsection{Analyses secondaires}

Pour décrire les deux contextes variables (quantificateurs et nombres cardinaux), nous avons procédé à deux types d'analyses secondaires. Dans un premier temps, nous avons distingué six types de quantificateurs : 1- les quantificateurs comptables (plusieurs, certains), 2- les quantificateurs ambigus (beaucoup, peu), qui peuvent aussi apparaître avec des compléments massiques, 3- les quantificateurs proportionnels nominaux (la plupart, la majorité), 4- les quantificateurs numéraux approximatifs (une dizaine, une centaine), 5- le quantificateur négatif aucun(e) et le quantificateur chacun(e). Nous avons aussi choisi de codifier ces deux quantificateurs séparément puisque ces quantificateurs permettraient la variation (Mélis 2003). Dans un deuxième temps, nous avons distingué les cardinaux pluriels (deux et plus) des cardinaux singuliers (un(e), l'un(e)). Le tableau 4 résume les analyses secondaires effectuées.

\begin{tabular}{|c|c|c|c|}
\hline \multicolumn{5}{|c|}{ Type de quantificateur } \\
\hline comptable & ambigu & proportionnel & approximatif \\
\hline aucun(e) & chacun(e) & & \\
\hline \multicolumn{3}{|c|}{ Type de nombre cardinal } \\
\hline \\
deux et plus & un(e), l'un(e) \\
\hline
\end{tabular}

Tableau 4: Analyses secondaires

\subsubsection{Résultat des analyses secondaires}

Le tableau 5 présente le résultat du découpage des quantificateurs en différentes catégories. Ce découpage des quantificateurs fait ressortir deux types de comportements. Nous avons d'une part, les quantificateurs 
aucun(e) et chacun(e) qui permettent la variation, et d'autre part, les autres quantificateurs qui ne tollèrent pas la variation.

\begin{tabular}{|l|l|l|l|l|l|l|}
\hline & \multicolumn{3}{|c|}{ Le Monde 2002 } & \multicolumn{3}{c|}{ Presse canadienne } \\
\hline & Entre & & Total & Entre & & Total \\
\hline Q comptables & 452 & 100 & 452 & 163 & 99,4 & 164 \\
\hline Q ambigus & 103 & 99 & 104 & 14 & 100 & 14 \\
\hline $\begin{array}{l}\text { nominaux } \\
\text { proportionnels }\end{array}$ & 375 & 100 & 375 & 82 & 100 & 82 \\
\hline $\begin{array}{l}\text { numéraux } \\
\text { approximatifs }\end{array}$ & 72 & 100 & 72 & 18 & 100 & 18 \\
\hline $\begin{array}{l}\text { aucun(e) } \\
\text { chacun(e) }\end{array}$ & 79 & 76,7 & 103 & 18 & 75 & 24 \\
\hline & 117 & 50,9 & 230 & 28 & 40,6 & 69 \\
\hline
\end{tabular}

Tableau 5 : Distribution des variantes de et d'entre en fonction du type de quantificateurs

On ne retrouve que deux exceptions dans les deux sous-corpus : dans le corpus Le Monde 2002, cette exception concerne le quantificateur ambigu beaucoup (11), alors que dans le corpus canadien, l'exception implique le quantificateur comptable certains (12) dans du discours rapporté.

(11) Après l'attentat manqué du 20 juillet 1944, auquel le Führer échappa à son quartier général de Wolfschanze, beaucoup d'eux furent exécutés.

Le Monde 2002

(12) «Nous avons malheureusement perdu plusieurs joueurs et certains d'eux étaient de très gros morceaux de l'organisation. Denis Gaudet (Rogersville), un ancien des Centennials de North Bay, et Owen Millea (Big Cove), un ancien des Foreurs de Val - d Or, sont partis jouer ailleurs . Tu ne remplaces pas des joueurs de leur calibre du jour au lendemain », a déclaré Poirier.

\section{L'Acadie Nouvelle}

Dans le cas des nombres cardinaux, nous avons distingué les cardinaux pluriels (deux et plus) des cardinaux singuliers (un(e), l'un(e), un(e) seul(e)/seul(e) un(e)). Cette distinction met elle aussi en évidence deux types de comportements : d'une part, les cardinaux pluriels (deux et plus) exigent la présence de la préposition entre; d'autre part, les cardinaux singuliers (un(e), l'un(e), un(e) seul(e)/seul(e) un(e)) présentent un comportement variable.

\begin{tabular}{|l|l|l|l|l|l|l|}
\hline & \multicolumn{3}{|c|}{ Le Monde 2002 } & \multicolumn{3}{c|}{ Presse canadienne } \\
\hline & Entre & & Total & Entre & & Total \\
\hline deux et + & 492 & 100 & 492 & 116 & 100 & 116 \\
\hline un $(e)$ & 311 & $37,4 \%$ & 832 & 53 & $39,3 \%$ & 135 \\
\hline & 803 & $60,6 \%$ & 1324 & 169 & $67,3 \%$ & 251 \\
\hline
\end{tabular}

Tableau 6 : Distribution des variantes de et d'entre en fonction du type de nombre cardinal 


\subsubsection{Analyses complémentaires}

Afin de cerner le contexte de variation dans le cas des cardinaux singuliers (un(e), l'un(e), un(e) $\operatorname{seul}(e) / \operatorname{seul}(e)$ un(e)), nous avons procédé à des analyses complémentaires. Le tableau 7 présente la distribution de ces formes. Dans les deux sous-corpus, la présence de l'adjectif seul(e) force la présence de la variante d'entre et la forme complexe l'un(e) permet la variation à des taux semblables.

\begin{tabular}{|l|l|l|l|l|l|l|}
\hline & \multicolumn{3}{|c|}{ Le Monde 2002 } & \multicolumn{3}{c|}{ Presse canadienne } \\
\hline & Entre & & Total & Entre & & Total \\
\hline $\begin{array}{l}\text { Un(e) } \\
\text { seul(e) }\end{array}$ & 14 & $100 \%$ & 14 & 1 & $100 \%$ & 1 \\
\hline L'un(e) & 297 & $36,4 \%$ & 815 & 43 & $36,8 \%$ & 117 \\
\hline Un(e) & 0 & $0 \%$ & 3 & 9 & $52,9 \%$ & 17 \\
\hline & 311 & $37,4 \%$ & 832 & 53 & $39,3 \%$ & 135 \\
\hline
\end{tabular}

Tableau 7 : Distribution des variantes de et d'entre en fonction du contexte syntaxique du numéral un(e)

Par ailleurs, seul le sous-corpus canadien permet la variation lorsque un(e) apparaît seul. Dans Le Monde 2002, un(e) n'apparaît jamais suivi de la variante complexe d'entre Le peu d'occurrences de cette forme dans le corpus français ne nous permet pas de conclure que la présence de la préposition entre est impossible dans ce contexte. Comme nous l'avons mentionné dans l'introduction, Mélis (2003) mentionne la possibilité de variation dans le cas du déterminant un. En fait, notre analyse de corpus vient confirmer l'observation de Mélis, à savoir que seuls aucun(e), $\operatorname{chacun}(e)$ et un(e) permettent la variation. Nous avons pu toutefois rafiner cette généralisation : dans le cas de un(e), la présence de l'adjectif $\operatorname{seul}(e)$ rend la présence de la préposition obligatoire. La section qui suit examine plus en détail les contextes qui permettent la variation.

\subsection{Le contexte variable}

Les résultats des analyses ci-dessus nous ont permis d'identifier trois déterminants qui permettent la variation: aucun(e), chacun(e) et l'un(e $)^{2}$. Ces résultats préliminaires ne nous permettent toutefois pas de déterminer a- si l'effet lexical est significatif, b- si la personne ou le genre peuvent influencer le choix de la variante. Nous avons donc classé ces trois déterminants en fonction du genre du déterminant (féminin ou masculin) et de la personne du pronom pluriel (première, deuxième, troisième). Nous avons par la suite soumis nos données à une analyse multivariée (à l'aide du logiciel Goldvarb Lion). Toutefois, avant de procéder, nous avons exclu les formes chacun et chacune d'entre nous/vous comme illustré en (13). $\mathrm{Au}$ nombre de cinq, ces contructions ne permettent pas la variation: les cinq formes présentent une utilisation systématique de la variante complexe d'entre, peu importe l'ordre des pronoms (que ce soit chacun et chacune ou chacune et chacun) ou la personne du pronom pluriel (limitée à nous ou vous dans notre corpus).

(13) Chacune et chacun d'entre vous, conscient de ses responsabilités, par un choix de liberté, a contribué, ce soir, à forger le destin de la France. Le Monde 2002

Le tableau 8 présente les résultats de l'analyse de régression multiple. Les résultats non significatifs apparaissent entre crochets. 


\begin{tabular}{|c|c|c|c|c|c|c|}
\hline & \multicolumn{3}{|c|}{$\begin{array}{c}\text { Le Monde } 2002 \\
\text { Input: } 0.429 \\
\text { Total N: } 1142 \\
\end{array}$} & \multicolumn{3}{|c|}{$\begin{array}{c}\text { Presse canadienne } \\
\text { Input: } 0.428 \\
\text { Total N: } 211 \\
\end{array}$} \\
\hline & FW & $\%$ & $\mathrm{~N}$ & FW & $\%$ & $\mathrm{~N}$ \\
\hline \multicolumn{7}{|l|}{ Déterminant } \\
\hline $\operatorname{Aucun}(e)$ & .812 & 76,7 & 103 & .809 & 76 & 25 \\
\hline Chacun(e) & .537 & 49,6 & 224 & .477 & 40,6 & 69 \\
\hline L'un(e) & .444 & 36,4 & 815 & .437 & 36,8 & 117 \\
\hline Ecart & 37 & & & 36 & & \\
\hline \multicolumn{7}{|l|}{ Personne } \\
\hline Deuxième & .774 & 75 & 8 & & {$[16,7]$} & [6] \\
\hline Première & .600 & 59,2 & 103 & & {$[42,9]$} & [35] \\
\hline Troisième & .487 & 40,7 & 1031 & & {$[43,5]$} & [170] \\
\hline Ecart & 29 & & & & & \\
\hline \multicolumn{7}{|l|}{ Genre } \\
\hline féminin & & {$[43,2]$} & {$[220]$} & & {$[44,7]$} & [47] \\
\hline masculin & & {$[42,5]$} & [922] & & {$[42,1]$} & [164] \\
\hline & $\begin{array}{r}\text { Log de } \\
\text { vraisemblance } \\
=-745.141 \\
\end{array}$ & & & $\begin{array}{r}\text { Log de } \\
\text { vraisemblance } \\
=-137,314 \\
\end{array}$ & & \\
\hline
\end{tabular}

Tableau 8: Régression logistique des facteurs linguistiques contribuant à la probabilité de la variante d'entre dans les constructions partitives permettant la variation.

L'analyse multivariée des facteurs linguistiques contribuant à la propobabilité de entre dans les contextes variables montre non seulement que l'effet lexical est significatif dans les deux sous-corpus, mais aussi qu'il existe des similarités frappantes dans cet effet lexical entre ces deux sous-corpus : aucun(e) favorise très fortement la présence de la variante d'entre, l'un(e) favorise faiblement l'absence de la variante d'entre et chacun(e) offre des résultats mixtes, favorisant très légèrement la présence de d'entre dans Le Monde, mais favorisant faiblement son absence dans le corpus de journaux canadiens.

L'analyse multivariée révèle aussi que la personne est un facteur significatif dans Le Monde mais non dans le corpus de journaux canadiens. Dans Le Monde, la deuxième personne (vous) favorise fortement la présence de la variante d'entre, la première personne (nous) favorise légèrement la présence de la variante d'entre, alors que l'effet est contraire dans le cas des pronoms de la troisième personne (elles ou ils). Dans le corpus de journaux canadiens, non seulement ce groupe de facteur n'est pas significatif, mais le taux d'emploi de la préposition entre y suit des tendances très différentes, la deuxième personne excluant ici la présence de la variante d'entre. Cette différence est d'autant plus surprenante que l'effet lexical est similaire dans les deux sous-corpus. Finalement, notons que le genre n'est significatif dans aucun des deux sous-corpus. 


\subsection{Résumé}

Cette étude de corpus a permis de distinguer les contextes qui exigent la présence de la préposition complexe d'entre et des contextes qui permettent la variation entre de et d'entre. Les contextes qui requièrent la forme d'entre sont: les démonstratifs, les nombres ordinaux, les pourcentages, les superlatifs, les mots QU-, les quantificateurs autres que aucun(e) et chacun(e), les nombres cardinaux au-

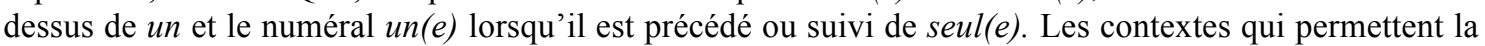
variation sont les quantificateurs aucun(e) et $\operatorname{chacun}(e)$, et les nombres cardinaux un(e) et l'un(e).

L'approche quantitative utilisée nous a permis d'établir la hiérarchie des facteurs dans les deux corpus journalistiques et de comparer les patrons de variation : non seulement les contextes variables et invariables sont les mêmes dans les deux sous-corpus, mais l'effet du facteur lexical est aussi le même dans les deux cas, le comportement du quantificateur aucun(e) se distinguant fortement des trois autres déterminants permettant la variation $(\operatorname{chacun}(e), u n(e)$ et l'un(e)).

Ces faits soulèvent plusieurs questions : 1- pourquoi le français exige-t-il la forme complexe d'entre dans les contextes invariables? Et qu'est-ce qui distingue les pronoms pluriels du français de ceux des autres langues romanes? 2- Pourquoi les déterminants aucun(e), chacun(e), un(e) et l'un(e) permettent-ils la variation entre de et d'entre? Et comment cette caractéristique distinctive rend-t-elle la présence de la forme complexe facultative ? 3- Existe-t-il d'autres mécanismes qui jouent le même rôle que entre dans les contructions partitives pronominales? La section qui suit propose quelques pistes d'analyse.

\section{Discussion}

\subsection{La forme complexe d'entre dans les constructions partitives pronominales}

Comme nous l'avons mentionné dans notre introduction, selon Franckel \& Paillard, les pronoms du pluriel désignent un ensemble non différencié et la préposition entre a pour fonction de partitionner l'ensemble dénoté par le pronom. Or, nous avons aussi vu que cette contrainte semble spécifique au français, les autres langues romanes ne nécessitant pas la présence d'une préposition comme entre. On peut donc se demander ce qui distingue les pronoms du pluriel en français des pronoms du pluriel des autres langues romanes.

De nombreuses études portent sur la pluralité et l'individuation (Chierchia 1998, Gillon 1992, Link 1983, Rothstein 2010, entre autres) et on distingue souvent deux classes morphosyntaxiques dénotant des ensembles d'entités comptables : les noms comptables et les collectifs. Les noms comptables ne dénotent pas d'ensembles d'entités de façon inhérente. En revanche, ils peuvent porter la marque morphologique du pluriel et leur projection étendue permet l'accord en nombre avec le prédicat.

(14) a. Le chat a mangé la souris.

b. Les chats ont mangé les souris.

La façon de marquer le pluriel n'est toutefois pas toujours la même et Bouchard (2002) distingue deux types de langues: alors que les langues comme l'anglais marquent le nombre (\#) sur N, le français marque le nombre sur D. Les pronoms pluriels partageant plusieurs des propriétés des noms comptables au pluriel (ils dénotent des ensembles d'entités et permettent l'accord pluriel avec le prédicat), on peut se demander si cette différence est à la source du comportement particulier des pronoms pluriels dans les constructions partitives.

Nous pouvons faire l'hypothèse que dans les langues où le nombre est marqué sur $\mathrm{N}$, les pronoms sont des têtes nominales marquées pour le nombre et seraient par conséquent associés à une structure de division (au sens de Borer 2005). Les langues où le nombre est marqué sur D sont différentes. Dans ces langues, les pronoms requièrent une stratégie d'individuation supplémentaire---comme la présence du morphème d'individuation (entre)—lorsque l'ensemble dénoté par le pronom doit être partitionné en ses parties atomiques. 
Lorsque l'individuation n'est pas requise (comme dans les constructions non partitives), la présence de entre n'est plus nécessaire. De même, lorsque le pronom pluriel reçoit une interprétation massique comme en (15a) où le quantificateur massique un peu de impose l'interprétation massique du pronom, la présence de entre n'est plus exigée et est même impossible dans ce contexte.

(15) a. Il y a un peu de nous dans cette analyse.

b. *Il y a un peu d'entre nous dans cette analyse.

L'interprétation massique est d'ailleurs privilégiée en (16a), comparativement à (16b).

(16) a. Le meilleur de nous = ce qu'il y a de meilleur en nous

b. Le meilleur d'entre nous = la personne la meilleure d'entre nous

Cette analyse rend compte du fait que, lorsque le pronom est suivi d'un nombre cardinal comme deux en (17), la présence de la préposition entre n'est plus requise. Dans ce contexte, le pronom se déplace de N à $\mathrm{D}$, où il peut être marqué pour le nombre et être associé à une structure de division ${ }^{3}$.

(17) le meilleur de nous deux. = la personne la meilleure d'entre nous deux

Ce contexte correspond d'ailleurs à la moitié des six exceptions que nous avions relevées à la section 3.2 et que nous reprenons ici en (18).

(18) a. Le meilleur nageur de nous deux

b. Lequel de nous trois

c. Duquel de vous trois

En résumé, nous avons attribué le comportement singulier des constructions partitives pronominales en français au fait que cette langue marque le nombre sur D plutôt que sur N. Deux stratégies permettent de sanctionner les structures partitives pronominales : l'ajout d'un morphème d'individuation (entre) ou la montée du pronom sous D. La prochaine section étend cette analyse aux déterminants qui permettent l'alternance entre de et d'entre : $\operatorname{aucun}(e)$, chacun(e) et l'un(e).

\subsection{Pourquoi aucun(e), chacun(e), un(e) et l'un(e)?}

\subsubsection{La question du nombre}

Notre étude porte sur les constructions partitives pronominales et impliquent donc nécessairement la partition de l'ensemble d'entités dénoté par le pronom pluriel. Les déterminants permettant la variation entre de et d'entre étant tous singuliers, on peut se demander si le nombre du déterminant joue un rôle particulier dans le choix de la variante, comme le propose le Wiktionnaire, branche francophone du projet Wiktionary, sous l'entrée D'ENTRE : «On peut utiliser $\underline{d e}$ si le mot précédent est singulier. » On retrouve d'ailleurs sur Wiktionnaire les exemples suivants à l'appui de la généralisation :

(19) a. L'un d'entre nous. L'un de nous.

b. Deux d'entre nous.

c. Aucun d'entre nous. Aucun de nous.

Le fait qu'il existe de nombreuses contructions au singulier qui exigent la forme complexe d'entre milite contre cette hypothèse. Tout d'abord, nous avons vu que le déterminant un(e) exigeait la préposition complexe lorsqu'il est précédé ou suivi de $\operatorname{seul}(e)$. Ensuite, plusieurs des déterminants qui exigent la forme complexe peuvent avoir une forme au singulier comme les démonstratifs celui et celle (20a), les superlatifs la plus et le plus (20b), les nombres ordinaux le premier, la deuxième, etc. (20c), les mots Qu$q u i$ et lequel/laquelle (20d), les déterminants proportionnels la majorité, la plupart (20e) et les fractions la moitié, un tiers (20f). À noter que dans tous ces contextes, le verbe s'accorde au singulier. 
(20) a. Sur ce sujet-là, les républicains ont, fondamentalement, la confiance du pays, et particulièrement celui d'entre eux qui siège à la Maison Blanche.

b. La plus célèbre d'entre elles, la comète de Halley, dont le noyau a été survolé en 1986 par la sonde européenne Giotto, est une vieille habituée de ces voyages dans la banlieue solaire qu'elle fréquente tous les soixante-seize ans, donnant l'étonnant spectacle, comme au début du siècle, de sa queue développée sur des dizaines de millions de kilomètres.

c. La première d'entre elles, évidente, est économique.

d. Lequel d' entre eux, pourtant, aurait risqué de parier un dollar que John Forbes Nash deviendrait, trente ans plus tard, une célébrité pour le grand public?

e. La majorité d'entre eux appartient, en tout ou partie, à la municipalité.

f. Un tiers d'entre eux a créé un site ou envisage de le faire.

\section{Le Monde 2002}

Les exemples en (20) montrent clairement que le nombre ne peut à lui seul expliquer le fait que seuls les déterminants aucun(e), chacun(e), un(e) et l'un(e) puissent apparaître avec l'une ou l'autre des deux variantes. La section qui suit explore l'idée que le statut exceptionnel de ces quatre déterminants tient au fait qu'ils partagent un même élément morphologique : un.

\subsubsection{Une approche morphologique?}

Contrairement aux autres nombres cardinaux deux, trois, quatre, etc., un a un statut ambigu en français. Il est à la fois nombre cardinal (21a), article indéfini singulier (21b) et pronom indéfini (21c). Dans ce dernier cas, il peut apparaître précédé d'un déterminant défini (22a et 23a) et même porter la marque du pluriel ((23b), mais pas (22b)).

(21) a. Combien d'animaux as-tu chez toi ? J'ai un chat.

b. J'ai vu une chatte tigrée.

c. Q : Combien d'étudiants sont venus te rencontrer hier?

$\mathrm{R}$ : Un est venu avant le lunch et un autre après.

(22) a. l'un de ceux que tu connais

b. *les uns de ceux que tu connais

(23) a. l'un l'autre

b. les uns les autres

Selon l'analyse de Kayne (2015), le déterminant one de l'anglais serait bimorphémique et impliquerait un classificateur en plus du déterminant indéfini one. Étendre cette analyse aux déterminants aucun(e), chacun(e), et l'un(e) permettrait de supposer que ces déterminants impliqueraient eux aussi un classificateur.

À première vue, les déterminants aucun(e), chacun(e), et l'un(e) apparaissent bimorphémiques en français : ils sont composés d'un même morphème un(e) précédé de chac-, auc-et l', respectivement. La présence d'un classificateur dans ces formes complexes pourrait expliquer que la présence du morphème d'individuation entre ne soit pas obligatoire dans le cas de ces déterminants. Ce classificateur pourrait soit le pronom indéfini un(e), soit un des préfixes auc-, chac- et $l$ '.

De nombreux arguments viennent soutenir la première hypothèse. D'une part, le fait que la forme simple un(e) ne requiert pas la présence de entre (voir tableau 7) montre bien que la présence d'un préfixe comme $a u c$-, chac- et $l$ 'n'est pas nécessaire à l'individuation. D'autre part, nous avons vu à la section 3.4 que, dans les deux corpus, aucun(e) se démarque de l'un(e) et chacun(e) en favorisant très fortement la présence de la variante d'entre. Cette différence entre $\operatorname{aucun}(e)$ d'une part et chacun(e) et l'un(e) d'autre part, pourrait tenir au fait que la forme aucun(e) est lexicalisée ${ }^{4}$. La structure interne de aucun(e) étant devenue opaque, le mot n'est plus décomposable en deux parties auc-et un(e). Par conséquent, aucun(e) n'impliquerait donc plus de classificateur, ce qui expliquerait que sa distribution s'approche de celle des quantificateurs monomorphémiques et exige la présence du morphème d'individuation entre. Finalement, selon cette analyse, la présence obligatoire de la variante complexe d'entre dans le cas de un(e) 
seul(e)/seul(e) un(e)) serait attribuable au fait que l'adjectif seul(e) impose une interprétation comme numéral de un(e).

La présence d'un morphème classificateur dans les formes aucun(e), chacun(e), l'un(e) et un(e) s'avère être une piste prometteuse : comme les classificateurs permettent l'individuation, cela expliquerait que la présence du morphème d'individuation entre ne soit pas obligatoire. Cependant, l'étude détaillée de la composition interne des déterminants $\operatorname{aucun}(e)$, chacun(e), et l'un(e) dépasse de beaucoup les limites du présent travail, dont l'objectif était avant tout d'identifier les contextes qui exigent la présence de la variante complexe d'entre et de décrire l'étendue de la variation.

\section{Conclusion}

Cet article a tenté d'identifier les facteurs qui déterminent l'emploi de la préposition complexe d'entre dans les constructions partitives pronominales. Pour ce faire, nous avons effectué une étude comparative de deux corpus de journaux : le corpus Le Monde 2002 et un corpus de journaux canadiens couvrant la même période. Notre première conclusion est qu'il faut distinguer les contextes qui exigent la présence de la préposition complexe d'entre et des contextes qui permettent la variation entre de et $d$ 'entre.

Les contextes qui exigent la variante complexe d'entre de façon (quasi-)obligatoire sont :

- les démonstratifs,

- les nombres ordinaux,

- les pourcentages,

- les superlatifs,

- les mots QU-,

- les quantificateurs autres que aucun(e) et chacun(e),

- les nombres cardinaux au-dessus de un

- le numéral un(e) lorsque modifié par seul(e).

Les contextes qui permettent la variation sont :

- les quantificateurs aucun(e) et chacun(e)

- les nombres cardinaux un(e) et l'un(e).

Dans le cas de ces derniers contextes, l'utilisation des outils informatiques développés en sociolinguistique variationniste nous a permis de révéler l'existence d'un effet lexical important : seul le quantificateur aucun(e) favorise fortement la présence de la variante complexe d'entre. L'analyse comparée des deux corpus journalistiques a montré la similarité des patrons de variation, ce qui appuie la thèse selon laquelle la variété standard écrite serait la même en France et au Canada.

En dernier lieu, nous avons tenté d'établir un parallélisme formel entre les contextes qui exigent la préposition complexe et les contextes qui permettent la variation. Nous avons repris l'idée mise en avant par Franckel \& Paillard, selon qui la préposition entre a pour fonction de partitionner l'ensemble dénoté par le pronom et l'avons étendu aux formes morphologiquement complexe aucun(e), chacun(e) et l'un(e). Dans ces formes, le pronom indéfini un(e) sanctionnerait une structure de division qui aurait pour effet de partitionner l'ensemble dénoté par le pronom.

Notre étude repose sur des corpus journalistiques. La langue écrite étant beaucoup plus rigide que la langue parlée, on peut se demander si l'étude de corpus oraux offrirait la même homogénéité et la même rigidité dans le cas des contextes invariables. Il serait particulièrement intéressant de comparer des corpus oraux entre eux pour voir si d'autres facteurs peuvent venir influencer le choix des variantes. Par exemple, dans son étude portant sur la variation entre pronoms forts simples (nous/vous/elles/eux) et pronoms forts complexes (nous-autres/vous-autres/eux-autres) en français montréalais, Blondeau (2011) a montré que le choix de la forme simple du pronom était favorisée par la préposition entre, ce qui pourrait laisser croire que le suffixe -autres peut, tout comme les nombres cardinaux en position postpronominale (nous deux), sanctionner la structure de division. 


\section{Références bibliographiques}

Corpus

Corpus Le Monde 2002. Sous-corpus du corpus Le Migou. http://olst.ling.umontreal.ca/?page id=54

Corpus de presse canadien. Sous-corpus du corpus Le Migou. http://olst.ling.umontreal.ca/?page id=54

Études

Blondeau, H. (2011) Cet « autres » qui nous distingue. Tendances communautaires et parcours individuels dans le système des pronoms en français québécois. Québec, Presses de l'Université Laval.

Borer, H. (2005) In name only. Structuring Sense, Volume I. Oxford: Oxford University Press.

Bouchard, D. (2002) Adjectives, Number and Interfaces: Why Languages Vary. Oxford: Elsevier.

Chierchia, G. (1998) Plurality of mass nouns and the notion of "semantic parameter." In Rothstein, S. (éd.) Events and grammar, Dordrecht: Kluwer, 53-104.

Franckel, J.-J. et D. Paillard. 2007. Grammaire des prépositions, Tome 1. Paris, Ophrys.

Gillon, B. (1992) Towards a common semantics for English count and mass nouns. Linguistics and Philosophy 15, $597-640$.

Kayne, R. (2015) “English One and Ones as Complex Determiners. Manuscrit. New York University.

Link, G. (1983) The logical analysis of plurals and mass terms: A lattice-theoretical approach. In Bäuerle, R., C. Schwarze et A. von Stechow (éd.) Meaning, use and interpretation of language, Berlin: de Gruyter, 302-323.

Mélis, L. (2003). La préposition en français. Paris : Editions Ophrys.

Poplack, S. et S.A. Tagliamonte (2001) African American English in the Diaspora: Tense and Aspect, Malden: Blackwell.

Rand, D. \& Sankoff, D. (1990) GoldVarb: A variable rule application for the Macintosh. Centre de recherches mathématiques, Université de Montréal.

Rothstein, S. (2010) Counting and the mass/count distinction, Journal of semantics $27: 343-397$.

Sankoff, D., S.A. Tagliamonte, et E. Smith (2005) Goldvarb X. Dept. of Linguistics, U of Toronto, Canada. http://individual.utoronto.ca/tagliamonte/Goldvarb/GV_index.htm.

Wictionnaire. (https://fr.wiktionary.org/wiki/d\%E2\%80\%99entre, consulté le 13 décembre 2015)

\footnotetext{
${ }^{1}$ Pour leurs commentaires et suggestions, je tiens à remercier tout particulièrement Anne Bertrand, Hélène Blondeau, David-Etienne Bouchard, Heather Burnett, Lucas Champollion, Amy Rose Deal, Rose-Marie Déchaine, Brendan Gillon, Heidi Harley, Sali Tagliamonte, ainsi que les participants au séminaire du mercredi de l'UQAM et au colloque Pronomes: Morfossintaxe, Semântica e Processamento (UFB, Brésil). Cette recherche a reçu l'appui financier du Petite subvention UdeM-CRSH, ainsi que du Conseil de recherche en sciences humaines du Canada (subvention \#430-2015-00497 : Variation et diglossie en français québécois).

${ }^{2}$ Nous n'avons pas inclus le déterminant un(e) dans les analyses multivarées en raison de l'absence de variation dans le corpus français et du faible nombre d'occurrences dans le corpus canadien.

${ }^{3}$ Un relecteur anonyme remarque que l'exemple en (17) peut aussi avec une lecture « ce qu'il y a de meilleur en nous deux ». Cette lecture n'est pas disponible en (16b), c'est-à-dire lorsque le pronom est précédé de la préposition entre. Cette différence est importante puisqu'elle montre que l'adjonction du numéral cardinal permet mais ne force pas la structure de division.

${ }^{4}$ Je remercie Thomas Leu pour cette suggestion.
} 